\title{
The Relationship Between Risk Attitude and Strength of Preference: A Test of Intrinsic Risk Attitude
}

\author{
Ale Smidts \\ Erasmus University, Rotterdam School of Management, P.O. Box 1738, 3000 DR Rotterdam, The Netherlands
}

\begin{abstract}
Tn a field study, the concept of intrinsic.risk attitude is investigated. Intrinsic risk attitude concerns the relationship between risk attitude, measured by the utility function $u(x)$, and strength of preference, measured by the value function $v(x)$. We study farmers' decision-making vis-à-vis price risk and obtain assessments of risk attitude and strength of preference in two consecutive years in a sample of 253 respondents. This design enables us to investigate the temporal stability of intrinsic risk attitude. Our findings show that risk attitude and strength of preference are two distinctive constructs. More specifically, the hypothesis of a linear relationship between $u(x)$ and $v(x)$ is clearly rejected in favor of an exponential relationship. This exponential relationship implies that our respondents exhibit a constant absolute intrinsic risk attitude for different price levels, during a time period. Differences between respondents in direction and degree of intrinsic risk attitude are substantial and the majority of the respondents are intrinsically risk seeking. No statistically significant change in intrinsic risk attitude could be detected between the two years of measurement. However, the correlation between measures across time appears to be low. In the discussion, issues for further research are identified.

(Decision Analysis; Utility Theory; Utility Assessment; Value Assessment; Relative Risk Aversion)
\end{abstract}

\section{Introduction}

A fundamental difference between the expected utility model of Bernoulli (Bernoulli 1738) and that of von Neumann and Morgenstern (1947) concerns the definition and measurement of the evaluation function. In the Bernoulli model, the value function $v(x)$ is measured in riskless conditions and encodes strength of preference for outcomes. To Bernoulli, the diminishing marginal value for outcomes explains why decision makers are risk averse. In the expected utility model of vNM, the utility function $u(x)$ is a probability indifference curve measured by means of lotteries without referring to intensity of satisfaction (Fishburn 1989). In this model, the curvature of $u(x)$ reflects risk attitude.

Recently, these two models have been linked through the concept of intrinsic risk attitude (Bell and Raiffa
1982, Dyer and Sarin 1982). ${ }^{1}$ The hypothesis of intrinsic risk attitude states that an individual's preference for risky choice alternatives is a combination of: (1) the strength of preference the individual feels for the outcomes, and (2) his attitude towards risk. Risk aversion (as indicated by $u(x)$ ) is thus seen as the effect of diminishing marginal value (indicated by $v(x)$ ) plus the aversion against the dispersion in subjective values (intrinsic risk aversion). The utility function is a transformation of the strength of preference function: $u(x)$ $=f(v(x))$ and the curvature of $u(x)$ that has traditionally been seen to reflect risk attitude, in this view thus

\footnotetext{
${ }^{1}$ Intrinsic risk attitude is labeled relative risk attitude by Dyer and Sarin (1982), as it is defined relative to the strength of preference function. Here we use the former term to prevent confusion with the PrattArrow coefficient of relative risk aversion.
} 
reflects risk attitude and strength of preference combined. In general the relationship between $u(x)$ and $v(x)$ is hypothesized to be nonlinear. In particular, an exponential relationship has been suggested.

The purpose of this study is to empirically investigate the relationship between $u(x)$ and $v(x)$. By studying the relationship, insight is gained into the relevance of the concept. First, because the influence of the value function is accounted for, intrinsic risk attitude is considered to be a more accurate descriptor of the 'true' risk attitude of a decision maker than the conventional measure of risk aversion based on $u(x)$. It has been suggested that it would therefore be fairly stable over time and less dependent upon the decision context or the attribute of the decision. For example, the same intrinsic risk attitude may operate for such diverse attributes as money and time (Bell and Raiffa 1982). However, this view of a stable, trait-like property has been questioned (Schoemaker 1992). In this study we examine the temporal stability of intrinsic risk attitude.

Second, the notion of intrinsic risk attitude would be very convenient in modeling multiattribute decision making under risk. If a stable transformation function between $u(x)$ and $v(x)$ exists, then a multiattribute model under certainty can be transformed into a model under risk with only a small number of extra assessments (Dyer and Sarin 1982, Currim and Sarin 1983). A number of authors have challenged the concept of intrinsic risk attitude by pointing out that, normatively, the relationship between $u(x)$ and $v(x)$ should be linear (Allais 1979, Sarin 1982). Von Winterfeldt and Edwards (1986, p. 211) also argue that the distinction between utility and value is spurious. If that viewpoint proves to be empirically correct, then an even greater practical advantage for modeling decision making would arise, because then the usual models and measurement techniques under certainty can be applied directly in the analysis of decision making under risk.

Although a few researchers have empirically examined the concept of intrinsic risk attitude, these studies have yielded mixed results and were limited in their methodology. As discussed below, the studies typically use small student samples and pay little attention to validity and reliability issues. Also, no experience exists in measuring and testing the concept in survey research, although intrinsic risk attitude is considered relevant for the analysis of consumer decision making under risk (Currim and Sarin 1983).

In this study, we attempt to overcome the methodological limitations of prior research by testing the notion of intrinsic risk attitude in a naturalistic setting. We use real economic decision makers, a real decision problem, a large sample size and a longitudinal design. The farmers in our sample $(n=253)$ are deciding on the yearly repeating problem of selling their potato crop under price risk. In the study, risk attitude $(u(x))$ and strength of preference $(v(x))$ are measured with two carefully selected techniques. By repeating the measurements for the same respondents, estimates of the stability of the measurements are obtained. Finally, the relationship between $u(x)$ and $v(x)$ is tested for linear, exponential and power functions.

Section 2 elaborates on the concept of intrinsic risk attitude and reviews the relevant empirical literature. The design of the study and the measurement techniques will be described in $\$ 3$. In $\$ 4$ major findings with each technique will be given, and in $\$ 5$ the test of intrinsic risk attitude is presented by fitting the functional relationship between $u(x)$ and $v(x)$ (an analysis per individual). The final section summarizes the major results and provides both some implications and suggestions for further research into the concept.

\section{The Concept of Intrinsic Risk Attitude}

Three kinds of arguments have been discussed in the literature on intrinsic risk attitude: no relationship between $u(x)$ and $v(x)$, an exponential relationship, and a linear relationship. Each view and its empirical support is briefly discussed below. The section concludes by pointing out the gaps in the existing empirical research.

In the original formulation of vNM expected utility theory, no relationship between $u(x)$ and $v(x)$ was conceived (Fishburn 1989). For example, Baumol (1958) and Keeney and Raiffa $(1976$, p. 150) point out that vNM utility functions are completely different from the economist's cardinal utility function and that knowing one implies very little about the other. Also, Fishburn (1970, p. 82) has argued that a utility function does not measure preference differences between outcomes. In this 
view then, the concept of intrinsic risk attitude is not meaningful.

When a relationship is considered, two plausible functional relationships between value and utility functions are offered (Keeney and Raiffa 1976, p. 330 332, Bell 1981, Bell and Raiffa 1982, Sinn 1985, Barron et al. 1984). Either the relationship is linear, $u(x)=a$ $+b v(x)(b>0)$, or it is described by an exponential function, $u(x)=a+b e^{-c v(x)}(b>0)$, for $c \neq 0$ where $c$ is the Pratt-Arrow coefficient of local intrinsic risk aversion: $-u^{\prime \prime}(v(x)) / u^{\prime}(v(x))$; both $u(x)$ and $v(x)$ are defined up to a positive linear transformation. The exponential function implies a constant absolute intrinsic risk attitude. If the relationship between the functions is linear, then the decision maker is said to be intrinsically risk neutral, which means that diminishing marginal value completely explains his preferences under risk. Otherwise, the decision maker is said to be intrinsically risk averse $(c>0)$ or intrinsically risk seeking $(c<0)$. Keeney and Raiffa (1976) and Bell and Raiffa (1982) arrive at the conclusion of either an exponential or a linear function by analyzing the logical relationships between multiattribute value and utility functions. In essence it is shown that if each outcome in a lottery is increased in such a way that the same quantity measured in value is added to each outcome, the certainty equivalent also increases by this same quantity of added value (constant absolute risk attitude on $v(x))$. Sinn (1985) arrives at that conclusion from psychophysics.

Krzysztofowicz (1983a, b) found empirical support for a nonlinear relationship between value and utility functions after analyzing data of 34 respondents (a mixed sample of students, experts, and scholars). An exponential function fit his data well. However, other functional specifications were not tested. He found no tendency towards one type of intrinsic risk attitude (averse or seeking). Significant differences between $u(x)$ and $v(x)$ were also found by Keller (1985) in a study of 12 graduate students providing risky and riskless judgments for three decision problems, resulting in 29 assessments of both $u(x)$ and $v(x)$. The study was inconclusive with respect to the specific functional relationship. The exponential relationship was confirmed for ten out of 29 cases, the power function in four cases, and the logarithmic function in three cases.
In a third view, the idea of intrinsic risk attitude is discarded by proposing a linear relationship between $u(x)$ and $v(x)$ as the sole possibility. Allais $(1953,1979)$, in particular, fiercely objects to the idea of intrinsic risk attitude and introduced his 'axiom of cardinal isovariation' to prove the equivalence of value and utility functions (Allais 1979). Sarin (1982) and, in a similar vein, Bouyssou and Vansnick (1988) also introduced a number of conditions that can prove that $u(x)$ and $v(x)$ should be linearly related.

Some studies support the view of a linear relationship. Fischer (1977), in a study concerning the selection of multiattribute job descriptions by ten graduate students, found no difference between $u(x)$ and $v(x)$ for five out of ten respondents and a nonlinear relationship for the other five respondents. In a re-analysis of the Fischer (1977) data, Barron et al. (1984) concluded that in most of the ten cases, a linear relationship between the utility and value function provided a good fit and that an exponential transformation improved very little on the linear relationship. McCord and de Neufville (1983) compared the measurement of a value function with the assessments of utility functions for 23 subjects (MIT-students and faculty) by applying different assessment methods for $u(x)$. In the within-subject analysis, it turned out that differences between the utility functions were of the same magnitude as differences between the value function and the utility function assessed by means of the certainty equivalent method with $50 / 50$ probabilities. From these findings, Von Winterfeldt and Edwards (1986, p. 213) conclude that error and method variance within value and utility measurement procedures may overshadow, to a great extent, the subtle theoretical distinctions between $u(x)$ and $v(x)$. As a consequence, they advise applying both riskless and risky measurement procedures in measuring the evaluation function and recommend that respondents be urged to resolve inconsistencies between the measurements as much as possible (we will return to this issue in \$6).

The findings presented above leave us with an unclear picture of the specific relationship between $u(x)$ and $v(x)$. A number of studies found no difference or, relative to the magnitude of errors, only small differences between the functions. Other studies clearly found significant differences between $u(x)$ and $v(x)$, but 
were inconclusive with respect to the specific functional relationship. With respect to the methodology it can be concluded that most of the studies conducted so far are small in sample size and often use possibly lowly involved students as subjects, who are confronted with fairly hypothetical choice alternatives. Also, the studies provide no information with respect to the stability of the concept. In the design of our study we tried to overcome these limitations.

\section{Methodology}

\subsection{Overview}

In face-to-face interviews, single-attribute value and utility functions were assessed for farmers concerning the price for potatoes (in cents per kilogram, denoted by cts $/ \mathrm{kg}$ ). The lottery technique was applied to measure risk attitude $(u(x))$, the midvalue splitting technique was used to measure strength of preference $(v(x))$. The measurements were repeated for the same respondents one year later.

\subsection{Decision Context}

The test of intrinsic risk attitude took place in the context of a larger study of decision making under risk concerning farmers coping with the problem of selling their potato crop vis-à-vis price risk (Smidts 1990). The farmer's risky decision is to decide when and how much to sell at the spot market and/or how much to sell by means of a particular type of contract. Potatoes are the main source of income for the family farms in this study. Usually a fourth to a third of the arable land is allocated to potatoes, which results in, on average, about 50 percent of farm net returns stemming from potatoes. At present, potatoes are also the most pricerisky crop. Prices fluctuate heavily, both from year to year, and within a marketing year (a marketing year runs from harvest in September up until the end of the storage period in June/July).

The farmers sell their harvest to wholesaling companies, which offer a number of selling options. A first option is to sell at spot market prices at one or several moments in the marketing year. About 40 percent of the total production is sold at the spot market. A second popular option, also with a market share of 40 percent, is the pooling contract in which the farmer receives ap- proximately the average spot market price. The farmer's price risk is minimal if he sells by means of a fixed-price contract (15 percent market share). Still another option is the bottom-price contract ( 5 percent market share). Typically, a farmer chooses a combination of selling options, analogous to a portfolio of stocks. For example, he can decide to sell 20 percent of his harvest at a fixedprice contract of $25 \mathrm{cts} / \mathrm{kg}$, sell 30 percent at a pooling contrart and sell the remaining 50 percent at the spot market at two moments in time. Of course, the choice of the fractions and the timing of the selling directly influence the expected price and the price risk of the portfolio.

\subsection{Data Collection}

In the questionnaire, information was gathered on $u(x)$ and $v(x)$, on price risk perceptions of portfolios (subjective probability distributions), on preferences for portfolios, on choice behavior, and on background variables. Because of the length of the questionnaire, the interview was divided into two parts with one week in between. A year later each respondent was interviewed again. However, because of budgetary constraints, only one interview per farmer was conducted in the second year. As a consequence the midvalue splitting technique was given to only a third of the respondents, randomly selected. The separate interviews lasted between 60 and 90 minutes.

Care was taken to minimize the interference of the measurement of $v(x)$ with that of $u(x)$. In the first year, $v(x)$ was measured in the first of the two interviews held with the respondents and $u(x)$ was assessed in the second interview. In the second year, the measurements were again maximally separated: $u(x)$ was measured in the first part of the interview and $v(x)$ towards the end.

A probability sample of $\mathbf{2 8 9}$ farms was drawn from 932 family farms in the main potato growing area of the Netherlands. The response rates were very high: 87.5 percent in the first year (253 farmers, of whom 15 refused cooperation in the second interview) and 81 percent (205) in the second year, indicating highly involved participants. Apart from item non-response, data for 205 respondents are available in both years of measurement. The average age of the farmer in the sample is 46 years, and most farmers are well educated. The interviewers were graduate students in agricultural econom- 
ics (average age was 25). All interviewers had prior interviewing experience and received extensive training in the assessment procedures.

\subsection{Measurement Procedures}

The attribute for which $u(x)$ and $v(x)$ were assessed is the price for potatoes in cts $/ \mathrm{kg}$, and this attribute ranged from 10 to $70 \mathrm{cts} / \mathrm{kg}$. The boundaries of the attribute reflect the minimum and maximum market prices; the 14-year averaged price is $24 \mathrm{cts} / \mathrm{kg}$. In all assessments, the farmer had to imagine selling his total potato harvest at the assessed prices.

The lottery or midpoint chaining technique (Farquhar 1984) was applied to measure $u(x)$. Respondents were confronted with 50/50 lotteries for which they had to state the certainty equivalent. To our respondents, this was a fairly realistic question because the certainty equivalent is comparable to a fixed-price contract, and the lottery can be viewed as selling on the spot market. The first certainty equivalent $x_{1}$ was assessed for the 50 / 50 lottery with the outcomes 10 and $70 \mathrm{cts} / \mathrm{kg}$ (expected utility of this lottery is 0.5 by scaling $u(10)=0$ and $u(70)$ $=1$ ). The certainty equivalent was arrived at by means of iteration. Subsequently, certainty equivalent $x_{2}$ was assessed for the 50/50 lottery $\left[10, x_{1}\right]$ and $x_{3}$ for the 50/ 50 lottery $\left[x_{1}, 70\right]$, with expected utilities of 0.25 and 0.75 , respectively. By appropriately chaining the lotteries, seven points of the utility curve were assessed, dividing the utility scale in eight equal parts of 0.125 utility (Keeney and Raiffa 1976). ${ }^{2}$ In the first year three consistency checks were assessed: two at $u(x)=0.50$ and one at $u(x)=0.375$. Thus, in total, ten points of the utility function were assessed. In the second year only one consistency check at $u(x)=0.5$ was assessed in order to reduce interviewing time. This was deemed justified because analysis in the first year had shown that respondents are highly consistent in their assessments. Per re-

\footnotetext{
${ }^{2} \mathrm{~A}$ consequence of the chaining procedure is the varying expected value and range of the lottery, which might induce biases (Krzysztofowicz and Duckstein 1980). However, a lottery played on the lower part of the price interval reflects the decision problem a farmer faces in a year in which the overall market price is fairly low, whereas lotteries with high outcomes reflect a year with a high overall price level. The varying expected value of the lottery thus adds realism to the assessment task.
}

spondent, both an exponential and a power function were fit to the data points. In Appendix A, the procedure applied in estimating the parameters is described.

In the second procedure, risk is not introduced into the measurement, so that a strength of preference function $v(x)$ is obtained. Fishburn (1967), von Winterfeldt and Edwards (1986), and Farquhar and Keller (1989) provide overviews of available techniques. We applied the midvalue splitting technique, or bisection technique (Torgerson 1958), which parallels the lottery technique with respect to the questioning procedure. The same boundaries ( 10 and $70 \mathrm{cts} / \mathrm{kg}$ ), sequence, and number of successive bisections were chosen (a consistency check at $v(x)=0.375$ was included, resulting in the assessment of eight points of $v(x))$. However, now the respondent had to state his equal-difference points instead of the certainty equivalents. On the interval $\left(x_{\mathrm{L}}\right.$, $\left.x_{H}\right)$, the equal-difference point $x_{E D}$ is the point for which the following relationship holds (Dyer and Sarin 1982): $v\left(x_{L}\right)-v\left(x_{E D}\right)=v\left(x_{E D}\right)-v\left(x_{H}\right)$. A respondent was asked to specify a price level $C$ between $A$ and $B$ $(A<C<B)$ so that the increase in price from $A$ to $C$ equals, in value, the increase from $C$ to $B$. The equaldifference point was arrived at by means of iteration. A number of test questions made the respondent familiar with the questioning procedure. According to Torgerson (1958) the midvalue splitting technique is one of the most simple scaling techniques because an ordinal judgment of the respondent suffices. By comparing two intervals, the respondent has to state only whether one interval is larger in subjective value than the other interval. The same procedure was followed in estimating the parameters in the exponential and power functions as with the lottery measurement (see Appendix A).

The two techniques described above enable a fairly stringent test of intrinsic risk attitude. Assessment techniques are subject to a host of response mode effects and procedural biases, see e.g. Hershey et al. (1982) and Farquhar (1984). In general, preferences appear to be highly contingent upon a variety of task, context, and individual difference factors (Payne et al. 1992). As noted above, response errors and method effects might even obscure true differences between value and utility functions (McCord and de Neufville 1983). One way to deal with this issue is by selecting techniques that differ solely on whether risky or riskless judgment is required. 
The lottery technique and the midvalue splitting technique resemble one another to such a large extent that in our view only risk differs between techniques. If there are significant differences between those techniques in both years of measurement, then these results provide a strong confirmation for the existence of intrinsic risk attitude. Other design features that increase the validity of the measurements are the high involvement of the decision makers confronted with realistic choice problems.

\section{Results of the Measurement of $u(x)$ and $v(x)$}

\subsection{Overview}

Table 1 summarizes the major findings obtained with both measurement techniques. In both years, the response percentages are high. The midvalue splitting technique (henceforth MVS-technique) shows the lowest response. This result is most likely explained by the fact that in both years this assessment was done at the end of the interview, which may have made some respondents less motivated to complete the question.

In the assessment procedures consistency checks were included. If respondents are consistent, the same certainty equivalent (equal-difference point) for the same level of $u(x)(v(x)$, respectively) should result aside from random response error. Differences between consistency assessments appeared to be small. The median MAPE (mean absolute percentage error) is 8.3 percent $(2 \mathrm{cts} / \mathrm{kg}$ ) in the first and 7.9 percent in the second year for the lottery technique (assessed at $u(x)=0.5$ ); for the MVS-technique (assessed at $v(x)=0.375$ ) these figures are 5.6 percent in the first and 5.3 percent $(1 \mathrm{cts} /$ $\mathrm{kg}$ ) in the second year. The MAPE is smaller than ten percent for 60 percent of the respondents in the lottery technique and 75 percent of those in the MVS-technique. Therefore, both techniques are judged high in internal consistency.

For both $u(x)$ and $v(x)$, the exponential function fit the data much better than the power function. For example, in Year 1 the average MSE (mean squared error) for $u(x)$ was 7.54 for the exponential and 17.76 for the power function. This difference in fit is largely significant $\left(t_{224}=12.6, p<0.001\right.$; pairwise test); the exponential function showed a better fit than the power function for 84 percent $(189 / 225)$ of the respondents. Moreover, for those respondents for whom the power function fit best, the difference in MSE between power and exponential function was small (on average 1.56), whereas for the other respondents, the fit of the power function was substantially worse compared to that of the exponential function (average difference is 12.46). Therefore, the exponential function fit the data at least about equally but often much better than the power function for almost all respondents. Likewise, for $v(x)$, the average MSE of the exponential function in Year 1 was 5.97 compared to 16.86 for the power function $\left(t_{221}\right.$ $=9.22, p<0.001)$. For 79 percent $(176 / 222)$ of the respondents the exponential function fit best.

The fit of the exponential function is also expressed in terms of MAE (mean absolute error) and $R$-square. For each respondent these indices are obtained. In Table 1 the median values of the sample are presented. Overall, from these figures we conclude that in both years the exponential function fit the data quite well. The fit of $v(x)$ seems to be somewhat better than that of $u(x)$.

The MVS-technique clearly indicates that almost all respondents show decreasing marginal value (a concave function), which is what one would expect if the respondents frame potato prices as gains. ${ }^{3}$ Also in the lottery technique the large majority of respondents is classified as risk averse (a concave function). The percentage of risk seeking respondents is low and it should be noted that for those respondents the degree of risk seeking is small. The standard deviation of the parameters in Table 1 indicate the diversity among respondents with respect to the degree of risk attitude and strength of preference.

\subsection{Stability of Risk Attitude and Strength of Preference}

Table 1 shows that the difference in average strength of preference between both years of measurement is

\footnotetext{
${ }^{3}$ It was not explicitly checked whether farmers frame all prices in the range between 10 and $70 \mathrm{cts} / \mathrm{kg}$ as gains. They may have framed price levels into gains and losses with respect to a reference point, such as the perceived cost per $\mathrm{kg}$. In that case, most farmers will probably perceive prices as gains because the interviews were held in the middle of the growing season (June), when most costs already have been made and therefore should be and probably will be treated by farmers as sunk costs.
} 
SMIDTS

Risk Attitude and Strength of Preference

Table 1 A Summary of Major Findings with Both Assessment Techniques

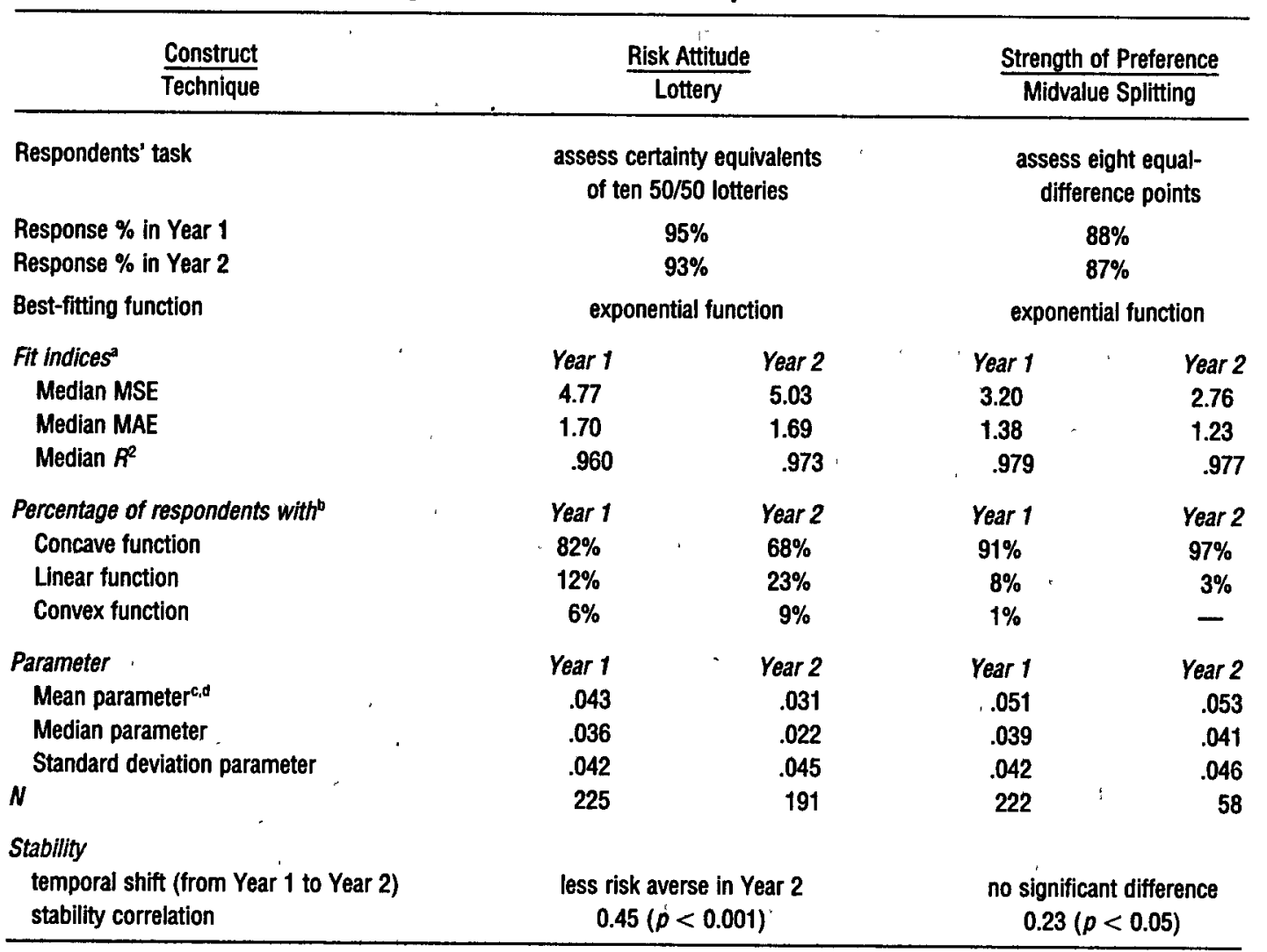

- MSE = Mean Squared Error, MAE = Mean Absolute Error; $R^{2}$ is calculated by squaring the Pearsoh correlation between the

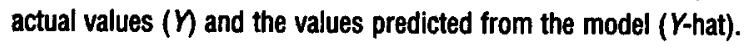

'Respondents are classified as having a linear function if the parameter is not significantly different from zero at the two-tailed $p=.10$ level (see Appendix A).

' The parameter reflects the Pratt-Arrow coefficient of absolute risk aversion (a larger positive parameter implies a more concave function).

d The parameter is estimated on the $[10,70]$ price interval ( $r$ in Equation A.2); multiply $r$ by 60 to obtain the parameter value on the normalized price interval $[0,1]$.

small. The shift in parameter value is not significant (pairwise $t$-test and Wilcoxon, $p<0.68$ ). However, with respect to the risk attitude a significant shift towards less risk aversion showed up in the second year (mean parameter estimates are: 0.043 in Year 1 and 0.031 in Year 2; pairwise tests, $p<0.001$ ). The parameter is smaller (indicating less risk aversion) in Year 2 than in Year 1 for 65 percent of the respondents. In particular the percentage of risk neutral respondents increased from 12 percent in Year 1 to 23 percent in Year 2. Because this topic was not explicitly researched, we can only speculate here about possible causes why such a shift may have occurred. It seems likely that farmers adapted their risk attitude to the different market circumstances. From the subjective probability distributions that were assessed, it was evident that farmers expected lower prices in Year 2 compared with Year 1. Also, their expectations about yield (in $\mathrm{kg}$ ) were lower. Both factors probably contributed to farmers' more pessimistic state of mind in the second year. Some farmers may even have perceived that they operated in the domain of losses. If so, this mind set may have induced them to become less risk averse in order to compensate for the 
expected decrease in wealth (Kahneman and Tversky 1979). ${ }^{4}$ After the fact, it indeed turned out that farmers were correct in their assessment of future prices: the actual potato prices in the second year were lower than in the first year; both years were below average.

Another aspect of stability is the test-retest correlation between the estimated parameters in the exponential function: The parameter obtained in the first year is correlated with the parameter obtained in the second year using the same technique. It indicates to what extent the rank order of respondents with respect to risk aversion in the first year corresponds with that rank order in the second year. The stability of the lottery technique is highest (Spearman $r=0.45$, Pearson $r=0.44, p<0.001$, $n=179$ ). In evaluating the magnitude of this correlation, note that the time span between the measurements is one year. This is an extremely long time interval for test-retest measurements. Schoemaker and Hershey (1992) report a test-retest correlation of $r=0.55$ for the CE-technique with a three-week interval. To our knowledge only in the study of MacCrimmon and Wehrung (1986), which concerns the risk attitudes of 84 business executives, were measurements repeated with a year in between. They found a test-retest correlation of $r=0.35$ $(p<0.001)$ for personal investments; the correlation was not significant for business investments. The stability in our study is good in comparison with their findings. The stability of the MVS-technique (Spearman $r$ $=0.24$, Pearson $r=0.23, p<0.05, n=55$ ) is fairly low compared to that of the lottery technique. Lack of comparable studies makes it difficult to judge the magnitude of this correlation. ${ }^{5}$

\footnotetext{
'The decrease in risk aversion also became apparent in the farmers' behavior. At the time of the interview, farmers were very reluctant to sell by means of the (low) fixed-price or bottom-price contract, adopting instead a 'wait and see' strategy.

${ }^{5}$ As the assessments took place a year apart, one cannot necessarily conclude from the fairly low stability correlations that the assessments are unreliable. Indeed, unreliability cannot be separated from attitude change with only two measurement occasions (Heise 1969). If attitudes did actually change, then even a perfectly reliable measurement instrument would yield low stability correlations. The estimated stabilities are therefore the lower bound of the test-retest reliability of each technique.
}

\subsection{The Relationship between Risk Attitude and Strength of Preference}

In an analysis across individuals, the relationship between risk attitude and strength of preference is established by correlating the parameter of the exponential function of $u(x)$ with that of $v(x)$. Risk attitude and strength of preference correlate positively and statistically significantly: in Year 1 the Pearson correlation is $r$ $=0.22(p<0.001, n=203)$, in Year 2 this correlation is somewhat higher: $r=0.33(p<0.014, n=53)$. The Spearman correlations are higher in both years, $r=0.26$ $(p<0.001)$ and $r=0.40(p<0.001)$, respectively. These significant correlations show that in this study risk attitude and strength of preference are not independent: a more concave $v(x)$ goes together with a more concave $u(x)$. On the other hand, the correlation is far from perfect. If risk attitude and strength of preference are one and the same construct $(u(x)$ and $v(x)$ are linearly related to one another or monotonically related with the same parameter for every subject), one would have found a much stronger, up to perfect, relationship. The low correlations thus indicate that $u(x)$ and $v(x)$ are not linearly related and that subjects differ in direction and/or degree of intrinsic risk attitude. From the findings of the analysis across respondents, it is concluded that the observed correlations tend to confirm rather than disconfirm the hypothesis of intrinsic risk attitude: risk attitude and strength of preference seem different constructs. In the next section a more definite test of the concept is provided by performing a detailed analysis of the relationship between $u(x)$ and $v(x)$ per individual.

\section{Test of Intrinsic Risk Attitude}

In an analysis per individual the specific functional relationship between $u(x)$ and $v(x)$ is tested. As was outlined in $\$ 2$, the exponential function has been proposed in describing the relationship between the value and utility function:

$$
u(x)=\frac{1-e^{-c v(x)}}{1-e^{-c}}
$$

with $0 \leq v(x) \leq 1$ and $0 \leq u(x) \leq 1$ and $c \neq 0$. The fit of this function will be compared to that of the linear model that serves as the benchmark (a linear relation- 
SMIDTS

Risk Attitude and Strength of Preference

Table 2 Results of Estimating Intrinsic Risk Attitude Per Individual, in Two Years (Sample Statistics)

\begin{tabular}{|c|c|c|c|c|}
\hline & \multicolumn{2}{|c|}{ Year $1(n=188)$} & \multicolumn{2}{|c|}{ Year $2(n=53)$} \\
\hline & Exponential & Power & Exponential & Power \\
\hline \multicolumn{5}{|l|}{ Parameter } \\
\hline Mean c & -.431 & 1.442 & -1.213 & 1.811 \\
\hline Median C & -.401 & 1.119 & -.696 & 1.266 \\
\hline Standard deviation c & 2.223 & 1.078 & 2.200 & 1.386 \\
\hline \multicolumn{5}{|l|}{ Fit indices ${ }^{b}$} \\
\hline Mean MSE & .0028 & .0038 & .0021 & .0034 \\
\hline Median MSE & .0012 & .0022 & .0017 & .0026 \\
\hline Mean $R^{2}$ & .974 & .970 & .983 & .978 \\
\hline Median $R^{2}$ & .989 & .985 & .988 & .980 \\
\hline Mean MAE & .038 & .046 & .035 & .047 \\
\hline Median MAE & .031 & .041 & .032 & .044 \\
\hline \multicolumn{5}{|l|}{ Percentiles parameter } \\
\hline 10th & -3.202 & .520 & -4.388 & .624 \\
\hline 25th & -1.550 & .799 & -2.498 & .995 \\
\hline 75th & .744 & 1.668 & -.026 & 2.257 \\
\hline 90th & 2.148 & 2.801 & 1.487 & 3.890 \\
\hline \multicolumn{5}{|l|}{$\begin{array}{l}\text { Classification of respondents: } \\
\text {-On the basis of parameter } \\
\text { value }^{c}\end{array}$} \\
\hline Intrinsically risk averse & $42 \%$ & $41 \%$ & $23 \%$ & $25 \%$ \\
\hline \multirow[t]{2}{*}{ Intrinsically risk seeking } & $58 \%$ & $59 \%$ & $77 \%$ & $75 \%$ \\
\hline & -On the basis of $t$-value & $100 \%$ & $100 \%$ & $100 \%$ \\
\hline Intrinsically risk averse & $32 \%$ & $33 \%$ & $23 \%$ & $23 \%$ \\
\hline Intrinsically risk neutral & $18 \%$ & $18 \%$ & $15 \%$ & $19 \%$ \\
\hline \multirow[t]{2}{*}{ Intrinsically risk seeking } & $50 \%$ & $49 \%$ & $62 \%$ & $58 \%$ \\
\hline & $100 \%$ & $100 \%$ & $100 \%$ & $100 \%$ \\
\hline
\end{tabular}

\footnotetext{
- The parameter estimates in this table reflect the Pratt-Arrow coefficients of intrinsic risk aversion; that is parameter $c$ in (5.1) and (5.2), respectively.

${ }^{\mathrm{b}} \mathrm{MSE}=$ Mean ,Squared Error; MAE = Mean Absolute Error.

' $A$ respondent is classified as intrinsically risk averse if $c>0$ (exponential function) or $c<1$ (power function); he/she is instrinsically risk seeking if $c<0$, or $c>1$, respectively.

- A respondent is classified as intrinsically risk neutral if the parameter is not significantly different from zero (exponential function) or one (power function) at the $\rho=.10$ level, i.e., if $-1.943<t$ $<1.943(d f=6)$.
}

ship between $u(x)$ and $v(x)$ implies that risk attitude and strength of preference are one and the same construct). The hypothesis of an exponential relationship can be substantiated further when it can be shown that the exponential function fits the data better than other reasonable nonlinear functions. Here we limit ourselves to a comparison of the fit of the exponential function to that of the power function, implying a decreasing absolute and constant proportional intrinsic attitude:

$$
u(x)=v(x)^{c}
$$


with $0 \leq v(x) \leq 1$ and $0 \leq u(x) \leq 1$. The parameter estimation procedure is described in Appendix $B$.

Descriptive statistics of the estimates are shown in Table 2. For each respondent (5.1) and (5.2) have been estimated; for the sample of respondents the mean and median parameters and fit indices (MSE, MAE and $R^{2}$ ) are presented. Respondents are classified in risk classes both with respect to the value of the parameter and on the basis of the $t$-value of the parameter.

First, it is found that both the exponential and the power function fit the data much better than the linear function. Mean MSE for the linear function is 0.033 in the first and 0.036 in the second year, which is eight to eighteen times as much as for the two nonlinear functions (see Table 2). For all respondents the linear function fits worse than the exponential or the power function. Moreover, the estimated parameters show the departure from the linear function: in both years the parameter of more than 80 percent of the respondents departs significantly from intrinsic risk neutrality ( $p<0.10$, two-tailed). We therefore reject the hypothesis of a linear relationship between $u(x)$ and $v(x)$.

Second, the fit indices in Table 2 indicate that, although both functions fit well, the exponential function fits the data better than the power function. Tested pairwise, the difference in MSE is largely significant in both years $\left(t_{187}=6.34, p<0.001\right.$ in Year 1 and $t_{52}=6.30, p$ $<0.001$ in Year 2). The fit of the exponential function is better than that of the power function for 74 percent of the respondents in Year 1 and 85 percent of the respondents in Year 2. It is concluded therefore that in this study a significant intrinsic risk attitude is found and that it is more likely to be a constant absolute intrinsic risk attitude (implied by an exponential function) than a constant proportional intrinsic risk attitude (implied by a power function). This finding confirms the hypothesis and supports and extends the findings of Krzysztofowicz (1983a, b) and Keller (1985).

Table 2 further indicates the general direction of the intrinsic risk attitude. The mean $c=-0.431$ in Year 1 and $c=-1.213$ in Year 2. These sample averages in both years imply that the average respondent exhibits intrinsically risk-seeking behavior. In the second year this tendency is larger than in the first year. Most of the farmers in our study are intrinsically risk seeking ( 50 percent in
Year 1 and 62 percent in Year 2). Krzysztofowicz (1983a, b) and Keller (1985) also found a high percentage of intrinsically risk-seeking respondents. If there is a tendency in the direction of intrinsic risk attitude, the sample mean of parameter $c$ should be significantly different from zero $\left(H_{0}: E(c)=0\right)$. Appropriate $t$-tests indicate that the tendency towards intrinsically risk-seeking behavior is significant in both years: $t_{187}=-2.66(p<0.01)$ and $t_{52}=-4.02(p<0.001){ }^{6}$ Such a tendency was not found by Krzysztofowicz (1983a, b).

Differences between respondents in intrinsic risk attitude appear to be large. The percentiles of the parameter distribution in Table 2 show that both strongly intrinsically risk seeking and strongly intrinsically risk averse respondents are present in the sample. Figure 1 illustrates these differences for both years.

The mean parameter values in Table 2 further suggest that in the second-year respondents became more intrinsically risk seeking. The percentage of intrinsically risk-neutral farmers remained about the same, whereas the percentage of intrinsically risk-seeking farmers increased from 50 percent to 62 percent. This finding is not surprising, because in Table 1 it was shown that $v(x)$ did not shift from Year 1 to Year 2, whereas $u(x)$ shifted significantly towards less risk aversion. The gap between $u(x)$ and $v(x)$ therefore widened for many respondents. However, the shift between the years is only marginally significant for the $n=48$ respondents for which the intrinsic risk parameter is obtained for both years: pairwise $t$-test (two-tailed), $t_{47}=-1.78$ $(p<0.081)$ and Wilcoxon, $z=-1.65(p<0.10){ }^{7}$ More specifically, 60 percent ( 29 out of 48 ) of the respondents became more intrinsically risk seeking, whereas 40 percent of the respondents became less intrinsically risk seeking. As a consequence the temporal stability correlation of intrinsic risk attitude, computed across respon-

\footnotetext{
${ }^{6}$ These tests may also be conducted with respect to the distribution of the $t$-values of the parameters (Nakanishi and Bettman 1974), so that the error in the estimate of the individual parameter is taken into account. In Year 1 the mean $t$-value is -1.33 (st. dev. is $11.05, n=188$ ); so $t_{187}=-1.65(p<.10)$. In Year 2 the mean $t$ is -3.49 (st. dev. is $10.68, n=53)$, so $t_{52}=-2.39(p<.02)$. The results parallel those obtained with respect to the parameter.

${ }^{7}$ Computed on the basis of $t$-values these test statistics are: $t_{47}=-1.53$ $(p<0.13)$ and Wilcoxon, $z=-1.19(p<.23)$.
} 
Figure 1 Estimated Intrinsic Risk Attitudes. Curves correspond to the 90th, 75th, 50th, 25th, and 10th percentile (from left to right, respectively); the 90th percentile in Year 1 indicates that $90 \%$ of the respondents' $c$ values falls below 2.15 .

Year 1

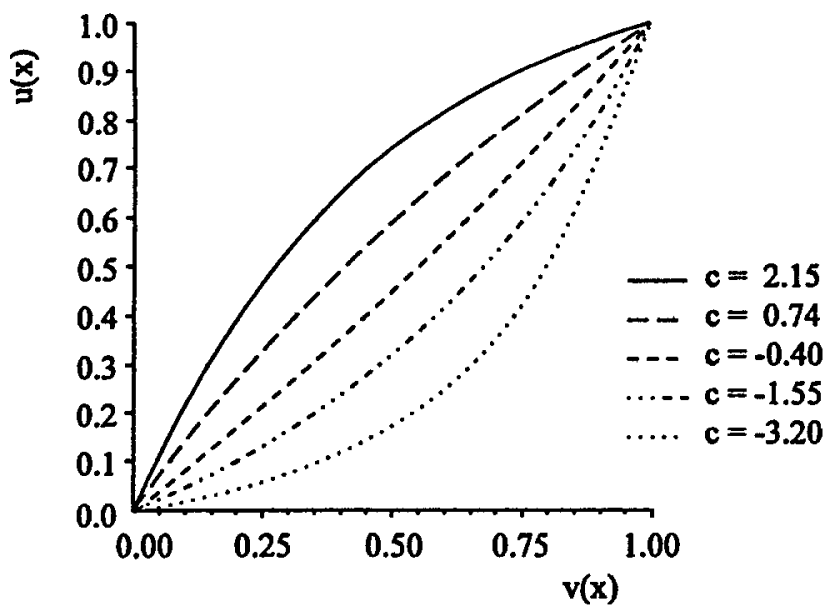

dents, is not significant: Pearson $r=0.10(p<0.50)$ and Spearman $r=0.15(p<0.32)$.

Finally, the fact that median values of the parameters are smaller than the mean values suggests that intrinsically risk-seeking respondents in both years exhibit their attitude more forcefully than those who are intrinsically risk averse (a skewed distribution). This tendency could not be statistically confirmed, however, because the Kolmogorov-Smirnov test does not reject the hypothesis of a normal distribution of the parameter at the one-tailed $p$-level of $0.10(p=0.32$ in Year 1 and $p$ $=0.104$ in Year 2).

\section{Discussion}

Before discussing further research, we summarize the major findings. The analysis supports the hypothesis that risk attitude and strength of preference are two distinctive constructs. More specifically, the hypothesis of a linear relationship between $u(x)$ and $v(x)$ is clearly rejected in favor of the theoretically proposed exponential relationship. In addition, the exponential relationship fits the data better than a power function. Respondents differ substantially in direction and degree of in-
Year 2

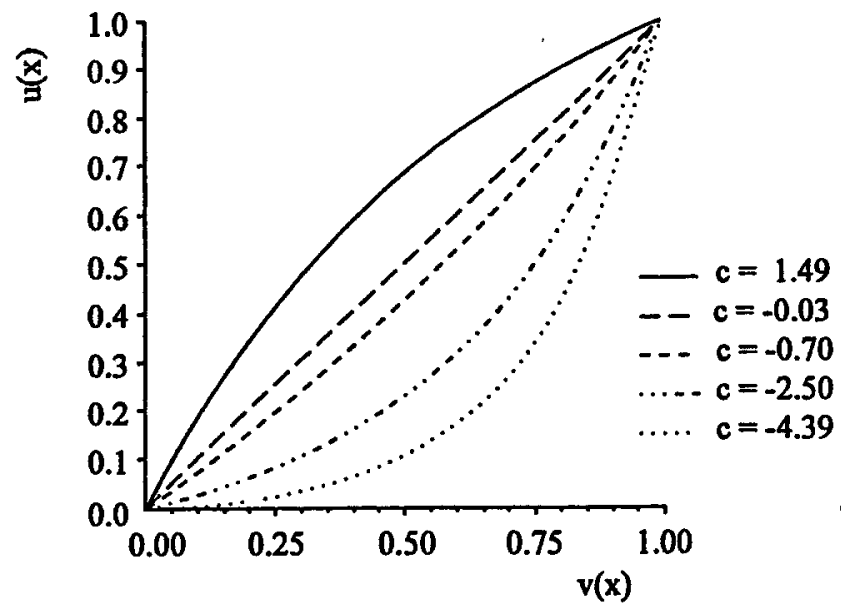

trinsic risk attitude. The majority of the respondents are intrinsically risk seeking. The tendency towards more intrinsic risk seeking between the years of measurement is not statistically significant.

The test of intrinsic risk attitude was conducted in a longitudinal and naturalistic setting with real decision makers and a real decision problem. These features of the study constitute a novel contribution to the existing, relatively small, empirical literature on the intrinsic risk attitude concept. For the first time, the temporal stability of intrinsic risk attitude was assessed.

In our study both procedures applied in measuring risk attitude and strength of preference yielded meaningful responses. We therefore strongly agree with von Winterfeldt and Edwards (1986) in recommending the use of multiple procedures, both risky and riskless, in assessing these constructs. However, we refute the idea of urging the respondent towards consistency between riskless and risky assessments, as they propose. In our view, the empirical evidence for intrinsic risk attitude from this and other studies is so strong that we believe that there exists a real difference between assessments under certainty and assessments under risk. 
The confirmation of intrinsic risk attitude in this study leads us to raise three issues for further research. First, does it predict choice behavior better? Second, is intrinsic risk attitude stable over time, attributes, and decision contexts? Third, what is the underlying psychological process that may explain intrinsic risk attitude?

First, one implication of finding a significant difference between $u(x)$ and $v(x)$ is that an expected utility model that incorporates $u(x)$ should have a higher predictive validity for risky choices than an analogous model that contains $v(x)$, since only $u(x)$ fully describes preferences under risk. Unfortunately, this hypothesis could not be adequately tested in our study. Several models were developed to predict the farmer's preferences for portfolios of selling options. The models incorporated the elicited subjective probability distributions for the portfolios and the respective evaluation functions. Due to a high percentage of second-degree stochastic dominance, prediction was fairly insensitive to the magnitude of the parameter in the evaluation function. More specifically, in about 50 percent of all pairwise comparisons of subjective probability distributions, the degree of risk aversion (or strength of preference) did not influence the preference order, because second degree of stochastic dominance existed. Consequently, $u(x)$ and $v(x)$ performed equally well in predictive validity (Smidts 1990). Clearly, further research into this issue is needed to adequately test predictive validity.

The second issue concerns the stability of intrinsic risk attitude. As stated earlier, it has been put forward that the relevance of the concept stems from the idea that the distinction between $u(x)$ and $v(x)$ clarifies choice behavior descriptively by disentangling the effects of both functions. In this manner a fundamental descriptor of the risk attitude of the decision maker can be found (stable over time, attributes and contexts). A problem with this assumption is that because intrinsic risk attitude is tied to the functions $u(x)$ and $v(x)$, only an indirect assessment of intrinsic risk attitude is possible. This implies that there are two sources of error in the assessment, which makes it vulnerable to instability. It is difficult to conceive of it as a more stable and fundamental characteristic of a decision maker if one does not at the same time conceive both $u(x)$ and $v(x)$ as equally stable. Empirical research by Keller (1985) and Krzysztofowicz (1983a, b) seems to indicate that intrinsic risk attitude is not invariant over decision attributes. This study showed the tendency to temporal instability over a single attribute. Compared to the first year, farmers adapted to the (perceived) change in market situation in the second year by changing their risk attitude $(u(x))$, whereas their valuation of the prices $(v(x))$ remained the same. On the positive side, it was also shown that in both years the exponential function described the relationship between $u(x)$ and $v(x)$ well. We therefore hypothesize that although the type of transformation function, specifically the exponential function, is stable over time (and perhaps, over attributes and context), the parameter in the function probably is not. Even in the latter case, however, multiattribute modelling of risky decisions can benefit from the concept of intrinsic risk attitude. Further research into the stability and context independence of intrinsic risk attitude is relevant.

As a final observation, this study showed that many decision makers are intrinsically risk seeking. The plausibility of intrinsically risk-seeking decision makers has not yet been addressed extensively. Moreover, the underlying psychological process explaining why some respondents become more cautious in the face of risk, whereas others do not, is unclear. Perhaps, as has been suggested by Weber (1994), configural-weight theory (Birnbaum et al. 1992) provides an avenue for theoretically clarifying the distinction between risk attitude and strength of preference. ${ }^{8}$

${ }^{8}$ The author gratefully acknowledges the contribution of professors Matthew Meulenberg and Berend Wierenga to this study. He would like to thank Robin Keller, Greg Fischer, two anonymous reviewers, Amna Kirmani, Paul Schoemaker, John Payne and Ben Bode for valuable and helpful comments on earlier drafts of the paper and Jaap Bijkerk for his technical assistance. The research was supported by grants from the Wageningen Agricultural University, the Rotterdam School of Management and the Netherlands Organization for Scientific Research.

\section{Appendix A}

Since the certainty equivalents for the 50/50 lotteries are measured with error, the inverse of the utility function is estimated (Knowles 1984):

$$
x_{1}=u^{-1}\left\{\frac{1}{2} u\left(x_{1}\right)+\frac{1}{2} u\left(x_{k}\right)\right\}+e_{3} .
$$


In (A.1), $x$, and $x_{k}$ respectively represent the low and high outcome in the 50/50 lottery and $x_{i}$ stands for the assessed certainty equivalent.

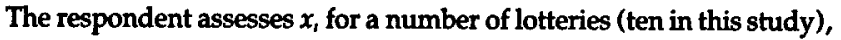
with varying outcomes $x_{j}$ and $x_{k} e_{i}$ indicates the response error. Assume the exponential utility function:

$$
u(x)=\frac{1-e^{-r\left(x-x_{L}\right)}}{1-e^{-r\left(x_{H}-x_{L}\right)}}
$$

with: $x_{L} \leq x \leq x_{H}$ and $0 \leq u(x) \leq 1, r \neq 0$. In this study $x_{L}=10 \mathrm{cts} /$ $\mathrm{kg}$ and $x_{H}=70 \mathrm{cts} / \mathrm{kg}$. In the exponential function, parameter $r$ is the Pratt-Arrow coefficient of absolute risk aversion (if $r>0$ the d.m. is risk averse, if $r<0$ the d.m. is risk seeking). Substituting (A.2) in (A.1) and rearranging yields:

$$
x_{1}=\frac{\ln \left\{\frac{1}{2}\left(e^{-r x_{i}}+e^{-r x_{k}}\right)\right\}}{-r}+e_{i}
$$

The least squares estimate of parameter $r$ in (A.3) is obtained by means of Fletcher's Quasi-Newton method (Maddala 1986). In a similar manner the parameter in the power utility function is estimated.

For classifying respondents into the broad categories of risk-averse, risk-neutral, and risk-seeking respondents, for each respondent it is tested by means of the $t$-test whether the parameter is significantly different from zero ( $p=0.10$, two-tailed) assuming that the errors $e_{\text {, }}$ are i.i.d. (Maddala 1986, p. 174).

\section{Appendix B}

The relationship between $u(x)$ and $v(x)$ is estimated per respondent. The MVS-technique yielded seven equal-difference points per respondent (the consistency check is excluded). The seven value levels $v\left(x_{i}\right)$ used to measure the equal-difference points $x_{1}$ are spread evenly over the interval between 0 and 1 with increments of 0.125 . The relationship between $u(x)$ and $v(x)$ is evaluated at these equal-difference points.

Specifically, for the exponential function, parameter $c$ is estimated in:

$$
u\left(x_{1}\right)=\frac{1-e^{-\cos \left(x_{i}\right)}}{1-e^{-c}}+e_{1}
$$

For the power function, parameter $c$ is estimated in:

$$
u\left(x_{1}\right)=v\left(x_{1}\right)^{c}+e_{1}
$$

with in both equations:

$u\left(x_{t}\right)=$ utility level of equal-difference point $x_{\text {, }}$ The utility levels $u\left(x_{1}\right)$ are calculated using the parameter of each respondent's own best fitting exponential function (i.e. using parameter $r$ estimated in (A.3); see Appendix A and Table 1),

$x_{1}=$ equal-difference point assessed by the respondent for value level $i$,

$v\left(x_{1}\right)=$ value level of equal-difference point $x_{1}$. That is, $v\left(x_{1}\right)=0.125$, $0.25,0.375,0.50,0.625,0.75$ and 0.875 , respectively,

$e_{t}=$ random error,

$i=1,2, \ldots, 7$,

$0 \leq u(x) \leq 1$ and $0 \leq v(x) \leq 1$.

Parameter $c$ in (B.1) and (B.2) is estimated by means of nonlinear least squares. For classifying respondents into risk classes of intrinsically risk averse, neutral or seeking, the same procedure as described in Appendix A has been applied.

\section{References}

Allais, M., "The Foundations of a Positive Theory of Choice Involving Risk and a Criticism of the Postulates and Axioms of the American School (1953)," in M. Allais and O. Hagen, eds., Expected Utility Hypothesis and the Allais Paradox, Reidel, Dordrecht, 1979, 27145.

, "The So-Called Allais Paradox and Rational Decisions under Uncertainty," in M. Allais and O. Hagen, eds., Expected Utility Hypothesis and the Allais Paradox, Reidel, Dordrecht, 1979, 437681.

Barron, F. H., D. von Winterfeldt, and G. W, Fischer, "Empirical and Theoretical Relationships between Value and Utility Functions," Acta Psychologica, 56 (1984), 233-244.

Baumol, W. J., "The Cardinal Utility which is Ordinal," Economic J., 68 (1958), 665-672.

Bell, D. E., "Components of Risk Aversion," in J. P. Brans, ed., Operational Research, North-Holland, Amsterdam, 1981, 371-378.

- and H. Raiffa, "Marginal Value and Intrinsic Risk Aversion," in H. Kunreuther, ed., Risk: A Seminar Series, IIASA, Laxenburg, Austria, 1982, 325-349.

Bernoulli, D., "Specimen Theoriae Novae de Mensura Sortis (1738)," translated as "Exposition of a New Theory on the Measurement of Risk," Econometrica, 22 (1954), 23-36.

Birnbaum, M. H., G. Coffey, B. A. Mellers, and R. Weiss, 'Utility Measurement: Configural-Weight Theory and the Judge's Point of View," J. Experimental Psychology: Human Perception and Performance, 18 (1992), 331-346.

Bouyssou, D. and J. C. Vansnick, "A Note on the Relationships between Utility and Value Functions," in B. R. Munier, ed., Risk, Decision and Rationality, Reidel, Dordrecht, 1988, 103-114.

Currim, I. S. and R. K. Sarin, "A Procedure For Measuring and Estimating Consumer Preferences Under Uncertainty," J. Marketing Res., 20 (1983), 249-256.

Dyer, J. S. and R. K. Sarin, "Relative Risk Aversion," Management Sci., 28 (1982), 875-886.

Farquhar, P. H., "Utility Assessment Methods," Management Sci., 30 (1984), 1283-1300.

- and L. R. Keller, "Preference Intensity Measurement," Ann. Oper. Res., 19 (1989), 205-217.

Fischer, G. W., "Convergent Validation of Decomposed Multiattribute Utility Assessment Procedures for Risky and Riskless Decisions," Organizational Behavior and Human Performance, 18 (1977), 295315.

Fishburn, P. C., "Methods of Estimating Additive Utilities," Management Sci., 13 (1967), 435-453.

- "Retrospective on the Utility Theory of von Neumann and Morgenstern," J. Risk and Uncertainty, 2 (1989), 127-158.

- Utility Theory for Decision Making, Wiley, New York, 1970.

Heise, D. R., "Problems in Path Analysis and Causal Inference," in E. F. Borgatta, ed., Sociological Methodology, Jossey-Bass, San Francisco, CA, 1969, 38-73. 
Hershey, J. C., H. C. Kunreuther, and P. J. H. Schoemaker, "Sources of Bias in Assessment Procedures for Utility Functions," Management Sci., 28 (1982), 936-954.

Kahneman, D. and A. Tversky, "Prospect Theory: An Analysis of Decision under Risk," Econometrica, 47 (1979), 263-291.

Keeney, R. L. and H. Raiffa, Decisions with Multiple Objectives: Preferences and Value Tradeoffs, Wiley, New York, 1976.

Keller, L. R., "An Empirical Investigation of Relative Risk Aversion," IEEE Trans. Systems, Man, and Cybernetics, 15 (1985), 475-482.

Knowles, G. J., "Some Econometric Problems in the Measurement of Utility," Amer. J. Agricultural Econ., 66 (1984), 505-510.

Krzysztofowicz, R., "Risk Attitude Hypotheses of Utility Theory," in B. P. Stigum and F. Wenstöp, eds., Foundations of Utility and Risk Theory with Applications, Reidel, Dordrecht, 1983b, 201-216.

- , "Strength of Preference and Risk Attitude in Utility Measurement," Organizational Behavior and Human Performance, 31 (1983a), 88-113.

- and L. Duckstein, "Assessment Errors in Multiattribute Utility Functions," Organizational Behavior and Human Performance, 26 (1980), 326-348.

MacCrimmon, K. R. and D. A. Wehrung, Taking Risks: The Management of Uncertainty, The Free Press, New York, 1986.

Maddala, G. S., Econometrics, McGraw-Hill, Singapore, 1986.

McCord, M. and R. de Neufville, "Empirical Demonstration that Expected Utility Decision Analysis is Not Operational," in B. P. Stigum and F. Wenstöp, eds., Foundations of Utility and Risk Theory with Applications, Reidel, Dordrecht, 1983, 181-199.

Nakanishi, M. and J. R. Bettman, "Attitude Models Revisited: An Individual Level Analysis," J. Consumer Res., 1 (1974), 16-21.
Neumann, J. von and O. Morgenstern, Theory of Games and Economic Behavior, 2d ed., Princeton University Press, Princeton, NJ, 1947.

Payne, J. W., J. R. Bettman, and E. J. Johnson, "Behavioral Decision Theory: A Constructive Processing Perspective," Ann. Rev. Psychology, 43 (1992), 87-131.

Sarin, R. K., "Strength of Preference and Risky Choice," Oper. Res., 30 (1982), 982-997.

Schoemaker, P. J. H., "Determinants of Risk-Taking: Behavioral and Economic Views," J. Risk and Uncertainty, 6 (1992), 47-74.

—and J.C. Hershey, "Utility Measurement: Signal, Noise and Bias," Organizational Behavior and Human Decision Processes, 52 (1992), 397-424.

Sinn, H. W., "Psychophysical Laws in Risk Theory," J. Econ. Psychology, 6 (1985), 185-206.

Smidts, A., Decision Making under Risk: A Study of Models and Measurement Procedures with Special Reference to the Farmer's Marketing Behavior, Dissertation, Wageningen Agricultural University, Pudoc, Wageningen, 1990.

Torgerson, W. S., The Theory and Measurement of Scaling, Wiley, New York, 1958.

Weber, E. U., "From Subjective Probabilities to Decision Weights: The Effect of Asymmetric Loss Functions on the Evaluation of Uncertain Outcomes and Events," Psychological Bulletin, 115 (1994), 228-242.

Winterfeldt, D. von and W. Edwards, Decision Analysis and Behavioral Research, Cambridge University Press, Cambridge, 1986.

Accepted by L. Robin Keller; received September 1, 1992. This paper has been with the author 14 months for 2 revisions. 